\title{
Implementation of Fuzzy Thresholding for Segmentation of Images
}

\author{
S. Santhi Kumari \\ Department of Electrical Engineering \\ University of Guyana \\ Turkeyen campus, Guyana, South America
}

\author{
Vamsidhar Enireddy, PhD \\ Department of CSE \\ WISTM Engg. College \\ Visakhapatnam, Andhra Pradesh, INDIA
}

\begin{abstract}
Segmentation is a process of dividing an image into multiple regions making it easier for the analysis. It is also an important step in Image processing. It also has number of applications which includes Medical field to analyze a disease, scientific fields including, engineering and technology, face recognition and object. Many algorithms and techniques have been developed for the image segmentation. To remove distinctive areas from a picture an immediate and straight forward method is using thresholding. It looks for a worldwide esteem that boosts the partition between yield classes. Noisy or uneven illuminated images pose a challenge for segmentation. The utilization of solitary hard limit esteem is absolutely the wellspring of imperative division mistakes in numerous situations like boisterous images or uneven illumination. In this paper a multiregion thresholding technique is displayed to overcome the normal downsides of thresholding strategies when images are debased with artifacts and commotion. Pixels from the images are related to various yield centroids by means of fuzzy membership function, evading any underlying hard choice. To make this strategy robust to noise and artifacts it utilizes spatial data through a local aggregation step where the participation level of every pixel is adjusted by neighborhood data that considers the enrollments of the encompassing pixels. The results are then compared with the existing techniques and results obtained are satisfactory.
\end{abstract}

\section{General Terms}

Image Processing, Segmentation.

\section{Keywords}

Image Processing, Segmentation, Thresholding, fuzzy.

\section{INTRODUCTION}

The primary aim of image processing is to recover required data from the given picture in a way that it won't impacts alternate highlights of those images and give the images that can be straightforward. De-noising of an image by utilizing channel is the most vital advance required to satisfy this prerequisite [1]-[2]. The undertaking of image segmentation is to isolate a picture into various non-overlapping locales. Which give same attributes like gray level, color, tone, texture [3]. The simplest segmentation method is thresholding. The pixels are partitioned depending on their intensity value. Image histogram helps to choose the suitable threshold value based on the peaks and valleys of the image.

Some factors affect the suitability of the histogram for guiding the choice of the threshold [4]:

(i). The separation between peaks;

(ii) The noise content in the image;
(iii)The relative size of objects and background;

(iv)The uniformity of the illumination;

(V)The uniformity of the reflectance.

Thresholding is a standout amongst the most immediate and basic ways to deal with image segmentation. It is an effective strategy as long as image indicates very much dense zones and the dark levels are bunched around far off qualities with least cover. It likewise has been utilized to give an underlying estimation or a preceding more perplexing division strategies (procedures in view of snakes, level-sets or dynamic shapes require an underlying division, that can be physically done or acquired by means of thresholding [5, 6]), to give veils of regions of interest [7], or, then again even as a method to distinguish movement in reconnaissance situations $[8,9]$. Thresholding is additionally broadly utilized as a part of the medical imaging field where images are created by a few tissues, represented by the gray levels [10]. The course of action of these tissues or organs inside the image is typically clearer than the arrangement of items in a natural scene image, consequently the utilizing of specific thresholding methods.

Otsu's method from the 70's is the most used thresholding technique in image segmentation [11, 12]. It searches for a global threshold value that separates classes in the result. This method is not effective in the case of noisy images, uneven illumination and soft transitions between the gray levels [13, 14 , and 15]. There are many approaches in the image segmentation methods that are well discussed in the literature $[13,16,17,18$, and 19]. The author in [13] has classified the thresholding methods mainly into six different categories as segmentation based on histogram $[11,12]$, Clustering based segmentation techniques[20,21, 22, 23, 24, 25], entropy based segmentation[26 27, 28], methods that extract the threshold value based on the features[29, 30], Based on the object attribute methods, using higher order probability distribution[15]. Histogram, clustering and entropy based segmentation methods are the foremost custom behind thresholding methods. Finding the optimal threshold is the main motto in the methods of the literature, still they depend on the gray level of pixel in the final stages of the segmentation. Many algorithms are proposed based fuzzy based techniques drop into these categories [31, 32, 33, 34, $35,36,37]$. These techniques fail when the images are noisy. When the key information is available about the objects in the images then the attribute based methods are a good source. In this paper a new method has been proposed considering and combining the features of adaptive local threshold and spatial local information for classifying the pixels. The membership degree of a specific pixel in a class is spatially related with the membership of its encompassing neighbors. The ultimate thresholding will consider the local membership in each of the classes, which implicitly makes the threshold locally variant. 
This method mainly relies on the Fuzzy Sets Theory and Fuzzy Logic [38].

It is a generalization of a classical logic developed by Lotfi Zadeh to model those problems in which imprecise data must be used or in which the rules of inference are formulated in a very general way making use of diffuse categories [39]. Fuzzy logic has much wide variety of applications in the real life. It is used in many engineering fields also. Control systems can be replaced with a fuzzy logic based systems [40]. Sendai Subway system in Sendai, Japan uses fuzzy logic controller to run the train [41] in image processing the fuzzy logic is used mainly in classification tasks when the features are not well defined. Information retrieval also uses fuzzy logic to achieve better results. A great progress has been made in the image processing applications [42]. In [42, 43] a great focus is done on applying fuzzy logic to the image thresholding in finding the optimum threshold without considering the spatial information.

\section{METHODOLOGY}

The main drawback of the existing techniques for the segmentation is corrupted images. The images can be corrupted by the noise and images with uneven illumination also create problems during the segmentation. The main disadvantage in using the global thresholds is it segments pixels with same intensity levels. To overcome this problem each pixel spatial surroundings has to be considered, then by the misclassification can be eliminated. The methodology is shown in the figure 1.

Consider that an image is defined as $\mathbf{I}(\mathbf{r})$ with $\mathrm{N}$ different regions and segmented image be $\mathbf{S}(\mathbf{r})$ which is obtained using thresholding. $\mathbf{S}(\mathbf{r})=\mathbf{m}_{\mathbf{a}}\{\mathbf{I}(\mathbf{r})\}$ Where $\mathbf{m}_{\mathbf{a}}$ is the method applied on the image for segmentation.

The input image is taken and it is segmented into different areas using $\mathrm{N}$ centroids. Available algorithms are used in obtaining the centroids. Entropy of histogram or clustering based can be used here.

A membership function can be defined as that specifies the degree to which a given input belongs to a set. The output is always limited to between 0 and 1 and it is known as membership value or grade. Many forms of membership functions are available in literature [43] such as Triangular. Trapezoidal. Piecewise linear. Gaussian. Singleton, etc., The only condition a membership function must really satisfy is that it must vary between 0 and 1 . There are 11 built in membership functions available in the Fuzzy logic toolbox.

The definition of the membership function can be done by from the histogram of the image or from the centroids, leaving aside histogram information [44]. To obtain a better solution Pseudo Trapezoid-Shaped (PTS) [45] membership functions is utilized. Membership is assigned to each pixel in the image. Spatial information is considered here to overcome the disadvantages in the previously stated algorithms in the literature. As fuzzy logic is applied, it creates many ways to reflect on the control of the neighborhood from already defined degrees.

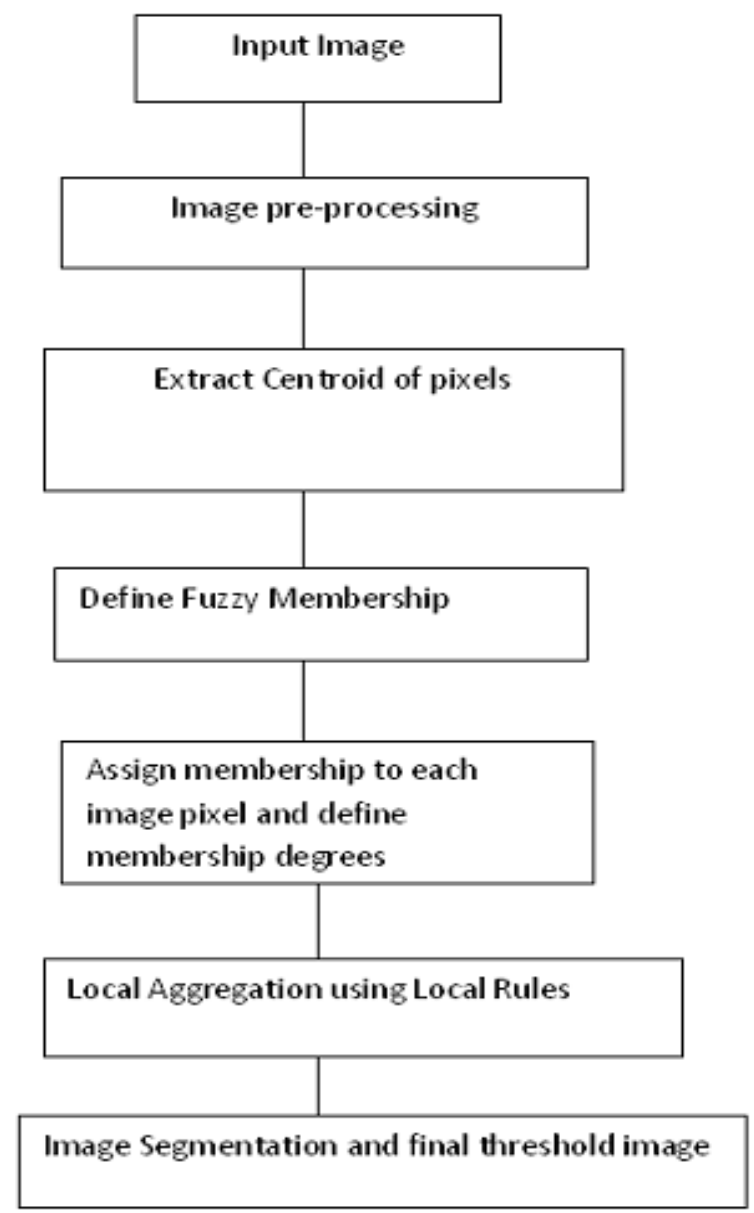

Fig 1: Figure showing the Methodology

A pixel is surrounded by a number of pixels around it so if we utilize the membership values of all pixels to classify the image into different regions. A local aggregation is defined as

$$
\mu^{S}(I(r))=\underset{S \in \varphi \in(r)}{a g g}\{\mu(I(s))\}
$$

Where agg $\{$.$\} is an aggregation in a neighborhood \varphi(r)$.

Using this function any modification can be done to the original membership functions whenever local information can be taken into account. Here effort is placed in the membership space and not over complete image. To get a better result, based on the pixels in the neighborhood diverse aggregations are defined. In [46, 47, 48, 49] authors have proposed various neighborhood based rule-sets for fuzzy image processing. Along with this we can also use the Median Aggregation, Average aggregation, Iterative averaging aggregation, Absolute Maximum.

In the final stage a segmented image can be obtained using the defined membership functions. Defuzzification can be used to obtained the output but a maximum operator [44]

$$
\left.M(O)=\operatorname{argmax}\left\{\mu_{l}^{S} I(r)\right)\right\}
$$

is used for obtaining final image. 


\section{EXPERIMENTS AND RESULTS}

In this paper three images were taken for testing the method and these images are shown in Fig.2. The images are obtained from The Berkeley Segmentation Dataset and Benchmark [59].

In order to apply the methodology on these images it is necessary to find out the influence of the centroid extraction on the final output. Histogram of the image I is calculated and the relevant maxima is considered and then the centroids are calculated from them. Now using this data the PTS membership functions are created from the centroids.

Using the equation

$$
\arg \min _{\theta(w)}\left|h(I)-\sum_{l=1}^{L} w_{l} \cdot p_{l}\left(x ; \theta_{l}\right)\right|^{2}
$$

where $\mathrm{h}(\mathrm{I})$ is the histogram of the image $\boldsymbol{p}_{\boldsymbol{l}}(\boldsymbol{x} ; \boldsymbol{\theta})$ a probability density function defined by the set of parameters $\boldsymbol{\theta}_{\boldsymbol{l}}$ and $\boldsymbol{w}_{\boldsymbol{l}}$ are weights satisfying that $\sum_{l} \boldsymbol{w}_{\boldsymbol{l}}=\mathbf{1}$, the centroids are obtained from the fitting of L Gaussian distributions to the histogram of the image $h$ (I) and Gaussian MF are created from the results.PTS membership functions are created using the centroids created by the centroids obtained from the fuzzy c-means [50].

The method is compared with some other techniques such as Otsu Thresholding method [51]. This method finds the threshold that minimizes the weighted within-class variance. Several assumptions are made during the approach where Histogram (and the image) is bimodal and also limited to no use of spatial coherence, nor any other notion of object structure.
Kmeans Clustering is an unsupervised clustering algorithm that minimizes the sum of square distances between all points and the cluster center. [52].

Fuzzy C-means clustering algorithm although introduced by Dunn [53] it was extended by Bezdek [54]. It is an iterative clustering method that produces an optimal c partition by minimizing the weighted within group sum of squared error objective function JF CM [54].Morphology based strategies utilize a calculation that computes the association cost between focuses in a image - that is, the smallest threshold that will provide a binary path between two points. These techniques have been utilized by $[55,56]$ and it is [56] has utilized it for the improvement of the airways in CT slices.

The measure for the comparison is made using the Jaccard similarity index [58], also known as the Tanimoto coefficient which measures the overlap of two sets [57].It is defined as the size of the intersection of the sets divided by the size of their union.

$$
\mathrm{J}(\mathrm{A}, \mathrm{B})=|\mathrm{A} \cap \mathrm{B}| /|\mathrm{A} \mathrm{U} \mathrm{B}|
$$

Where $A$ and $B$ are the two sets.

This can also be expressed in terms of the true positive (TP), false positive (FP), and false negative (FN) sets as TP / $(\mathrm{FP}+\mathrm{TP}+\mathrm{FN})[57]$.

The Jaccard index is zero if the two sets are disjoint, i.e., they have no common members, and are one if they are identical.

Higher numbers indicate better agreement in the sets. The results obtained are tabulated in table 2 which shows the different Jaccard index obtained for the images used in the experiment.
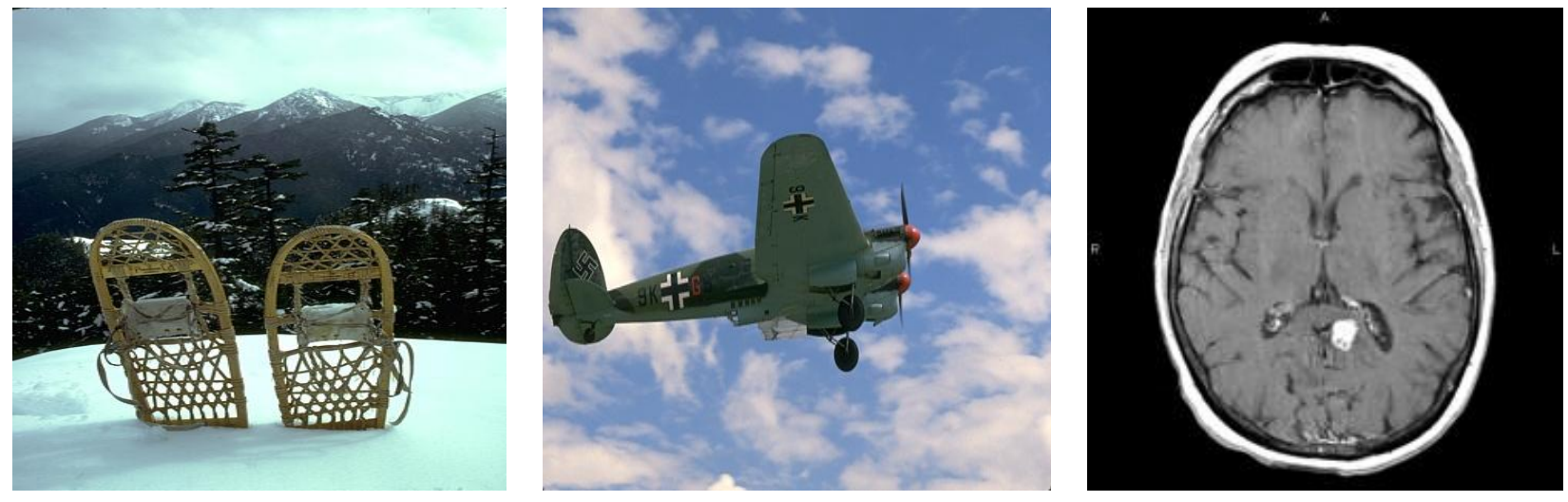

Fig 2: Images Used in the Experiment 
Table 1. Table captions should be placed above the table

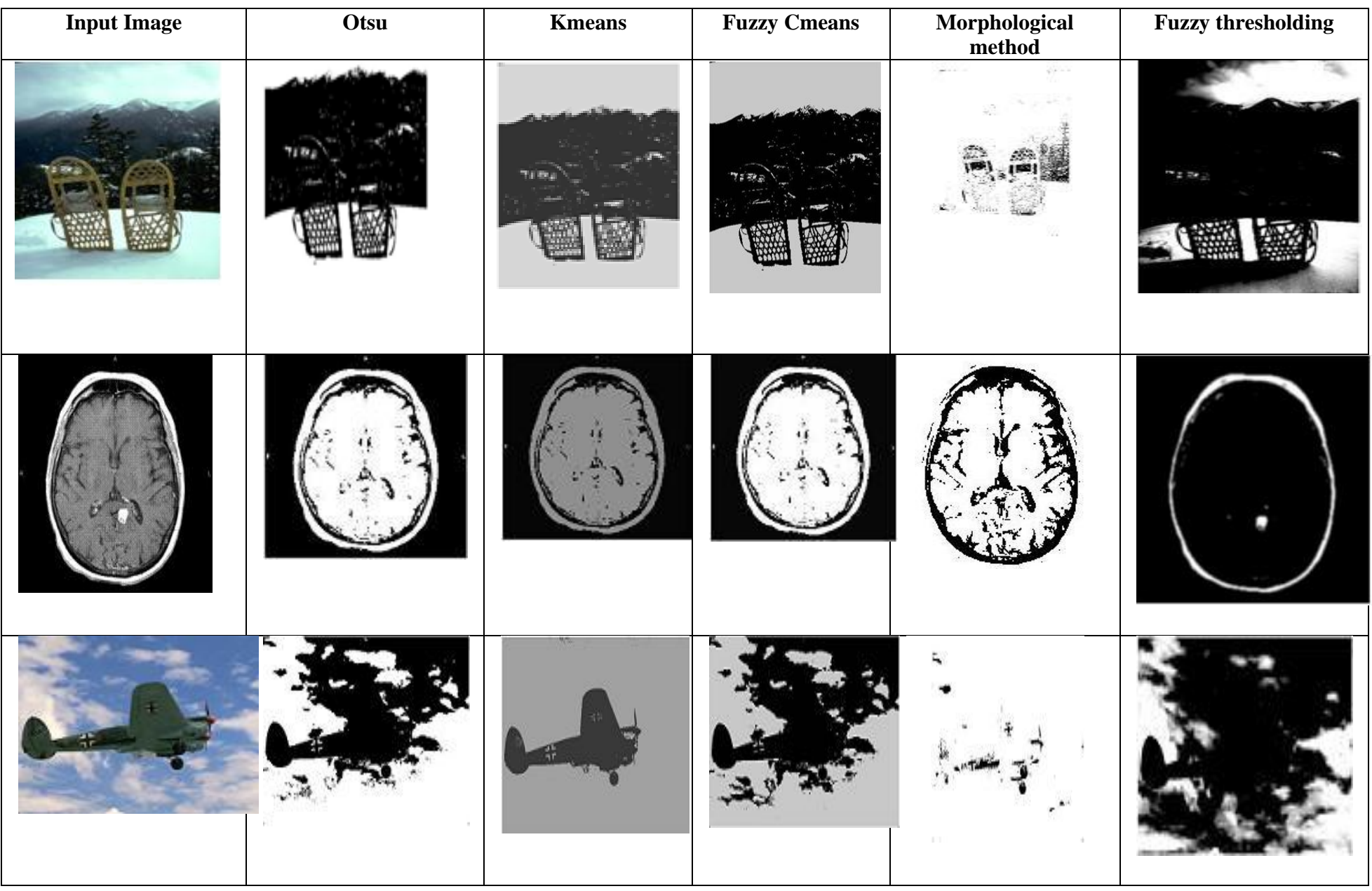

Table 2. Table showing the Jaccard index of the different images used in experiment

\begin{tabular}{|c|c|c|c|c|c|}
\hline $\begin{array}{c}\text { Input } \\
\text { Image }\end{array}$ & Otsu & Kmeans & Fuzzy Cmeans & $\begin{array}{c}\text { Morphological } \\
\text { method }\end{array}$ & $\begin{array}{c}\text { Fuzzy } \\
\text { thresholding }\end{array}$ \\
\hline 1 & 0.3086 & 0.6367 & 0.5234 & 0.3007 & 1.000 \\
\hline 2 & 0.3007 & 0.5703 & 0.5078 & 0.2812 & 1.000 \\
\hline 3 & 0.3086 & 0.6523 & 0.5273 & 0.3046 & 1.000 \\
\hline
\end{tabular}

\section{CONCLUSION}

The presented method had overcome many draw backs of the previous methods that are generally used in the segmentation process.FCM are used calculating the centroids which gives better results. The method is tested with three images and results obtained are satisfactory. The Otsu Thresholding, Kmeans Clustering method, Fuzzy C-means clustering method and morphological filtering and reconstruction. Methods are used for comparison. The Performance of the Fuzzy Thresholding method is better and it is compared using the Jaccard similarity index. In further study can be made on developing the membership function to increase the performance of the Fuzzy system.

\section{REFERENCES}

[1] M. Sharif, S. Mohsin , M. J. Jamal, and M. Raza, "Illumination normalization preprocessing for face recognition," in Proc .International Conference on Environmental science and information application technology ,pp. 44-47,2010.

[2] M. Yasmin, M. Sharif, S. Masood, M. Raza, and S. Mohsin, "Brain image enhancement-A survey," World Applied Sciences journal, , vol. 17, pp. 1192-1204, 2012

[3] J. Reinhardt and W. Higgins, "Automatis generation of image segmentation processes," IEEE Trans. Image Processing, , vol. 3, pp. 791-795, Nov 1994.

[4] http://homes.di.unimi.it/ferrari/ImgProc2011_12/EI2011 _12_16_segmentation_double.pdf 
[5] S. Taheri, S. Ong, V. Chong, Level-set segmentation of brain tumors using a threshold-based speed function, Image and Vision Computing 28 (1) (2010) 26 - 37.

[6] D. Mukherjee, N. Ray, S. Acton, Level set analysis for leukocyte detection and tracking, IEEE Trans.Image Proccess. 13 (4) (2004) 562-572.

[7] J. Sijbers, A. Postnov, Reduction of ring artefacts in high resolution micro-CT reconstructions, Physics in Medicine and Biology 49 (14) (2004) 247.

[8] P. L. Rosin, Thresholding for change detection, Computer Vision and Image Understanding 86 (2) (2002) 79 - 95.

[9] P. L. Rosin, E. Ioannidis, Evaluation of global image thresholding for change detection, Pattern Recognition Letters 24 (14) (2003) $2345\{2356$.

[10] G. Vegas-Sanchez-Ferrero, S. Aja-Fernandez, C. Palencia, M. Martin-Fernandez, A generalized gamma mixture model for ultrasonic tissue characterization, Computational and mathematical methods in medicine 2012.

[11] N. Otsu, A threshold selection method from gray-scale histogram, IEEE Trans. on Sys., Man and Cyb.9 (1) (1979) 62-66.

[12] T. Kurita, N. Otsu, N. Abdelmalek, Maximum likelihood thresholding based on population mixture models, Pattern Recognition 25 (10) (1992) 1231-1240.

[13] M. Sezgin, B. Sankur, Survey over image thresholding techniques and quantitative performance evaluation, Journal of Electronic Imaging 13 (1) (2004) 146-165.

[14] H. Wu, J. Barba, J. Gil, Iterative thresholding for segmentation of cells from noisy images, Journal of Microscopy 197 (3) (2000) 296-304.

[15] C. Leung, F. Lam, Maximum a posteriori spatial probability segmentation, Vision, Image and Signal Processing, IEE Proceedings- 144 (3) (1997) 161-167.

[16] P. K. Sahoo, S. Soltani, A. K. C.Wong, A survey of thresholding techniques, Computer Vision, Graphics, and Image Processing 41 (2) (1988) 233 - 260.

[17] R. C. Gonzalez, R. E. Woods, Digital Image Processing, Addision Wesley, 1992.

[18] L. Mahmoudi, A. El Zaart, A survey of entropy image thresholding techniques, in: Advances in Computational Tools for Engineering Applications (ACTEA), 2012 2nd International Conference on, IEEE,2012, pp. 204-209

[19] S. U. Lee, S. Yoon Chung, R. H. Park, A comparative performance study of several global thresholding techniques for segmentation, Computer Vision, Graphics, and Image Processing 52 (2) (1990) 171-190.

[20] F. R. D. Velasco, Thresholding using the isodata clustering algorithm, IEEE Trans. on Sys., Man and Cyb. SMC-10 (1980) 771- 774.

[21] H. Lee, R. H. Park, Comments on an optimal threshold schemefor image segmentation, IEEE Trans. On Sys., Man and Cyb. SMC-20 (1990) 741-742.

[22] W. Cai, S. Chen, D. Zhang, Fast and robust fuzzy cmeans clustering algorithms incorporating local information for image segmentation, Pattern Recognition 40 (3) (2007) 825-838

[23] Y. Yong, Z. Chongxun, L. Pan, A novel fuzzy c-means clustering algorithm for image thresholding, Measurement Science Review 4 (1) (2004) 11-19.

[24] A. Masood, Al-Jumaily, A. Ali, Fuzzy c mean thresholding based level set for automated segmentation of skin lesions, Journal of Signal and Information Processing 4 (2013) 66.

[25] B. N. Li, C. K. Chui, S. Chang, S. H. Ong, Integrating spatial fuzzy clustering with level set methods for automated medical image segmentation, Computers in Biology and Medicine 41 (1) (2011) 1- 10.

[26] T. Pun, A new method for gray-level picture threshold using theentropy of the histogram, Signal Processing 23 (1980) 223-237.

[27] T. Pun, Entropic thresholding: A new approach, Computer Graphics and Image Processing 16 (1981) 210-239.

[28] J. N. Kapur, P. K. Sahoo, A. K. C. Wong, A new method for gray-level picture thresholding using the entropy of the histogram, Graph. Models Image Process. 29 (1985) 273-285.

[29] L. Hertz, R. W. Schafer, Multilevel thresholding using edge matching, Comput. Vis. Graph. Image Process. 44 (1988) 279- 295

[30] L. OGorman, Binarization and multithresholding of document images using connectivity, Graph. Models Image Process. 56 (1994) 494-506.

[31] S. K. Pal, R. A. King, A. A. Hashim, Automatic gray level thresholding through index of fuzziness and entropy, Pattern Recogn. Lett. 1 (1980) 141-146.

[32] C. A. Murthy, S. K. Pal, Fuzzy thresholding: A mathematical framework, bound functions and weighted moving average technique, Pattern Recogn. Lett. 11 (1990) 197-206.

[33] K. Ramar, S. Arunigam, S. N. Sivanandam, L. Ganesan, , D. Manimegalai, Quantitative fuzzy measures for threshold selection, Pattern Recogn. Lett. 21 (2000) 1-7.

[34] O. J. Tobias, R. Seara, Image segmentation by histogram thresholding using fuzzy sets, IEEE Trans.Image Proccess. 11 (12) (2002) 1457-1465.

[35] M. Forero, Fuzzy thresholding and histogram analysis, in: M. Nachtegael, D. Van der Weken, D. Van deVille, E. Kerre (Eds.), Fuzzy Filters for Image Processing, Springer, Berlin, 2003, pp. 129-152.

[36] A. Z. Arin, A. F. Heddyanna, H. Studiawan, Ultrafuzziness optimization based on type ii fuzzy sets for image thresholding, ITB Journal of Information and Communication Technology 4 (2) (2010) 79-94.

[37] H. R. Tizhoosh, Image thresholding using type II fuzzy sets, Pattern recognition 38 (12) (2005) 2363-2372.

[38] J. M. Mendel, Fuzzy logic systems for engineering: A tutorial, Proceedings of the IEEE 83 (3) (1995) 345-377.

[39] L. A. Zadeh, Fuzzy logic and approximate reasoning, September 1975, Volume 30, Issue 3-4, pp 407-428. 
[40] J. M. Mendel, Fuzzy logic systems for engineering: A tutorial, Proceedings of the IEEE 83 (3) (1995) 345-377.

[41] http://sipi.usc.edu/ kosko/Scientific\%20American.pdf

[42] E. Kerre, M. Nachtegael (Eds.), Fuzzy techniques in image processing, Physica-Verlag, New York, 2000.

[43] http://www.philadelphia.edu.jo/academics/qhamarsheh/u ploads/Lecture\%2018_Different\%20Types\%20of\%20Me mbership\%20Functions\%201.pdf

[44] Santiago Aja-Fernandez, Ariel Hernan Curiale, Gonzalo Vegas-Sanchez-Ferrero, A local fuzzy thresholding methodology for multiregion image segmentation, Knowledge-Based Systems, January 26, 2015.

[45] X. Zeng, M. Singh, Approximation theory of fuzzy systems- SISO case, IEEE Trans. Fuzzy Sys. 2 (2) (1994) $162\{176$.

[46] F. Russo, fFIREg operators for image processing, Fuzzy Sets and Systems 103 (2) (1999) $265\{275$.

[47] H. Tizhoosh, Fast fuzzy edge detection, in: Fuzzy Information Processing Society, 2002. Proceedings.NAFIPS. 2002 Annual Meeting of the North American, 2002, pp. 239-242.

[48] D. Van De Ville, M. Nachtegael, D. Van der Weken, E. Kerre, W. Philips, I. Lemahieu, Noise reduction by fuzzy image filtering, IEEE Trans. Fuzzy Sys. 11 (4) (2003) 429-436.

[49] K.-S. Chuang, H.-L. Tzeng, S. Chen, J. Wu, T.-J. Chen, Fuzzy c-means clustering with spatial

[50] information for image segmentation, Computerized Medical Imaging and Graphics 30 (1) (2006) 9 -15.
[51] Yong Yang and Shuying Huang - 'Image Segmentation By Fuzzy C-Means Clustering Algorithm With A Novel Penalty Term" Computing And Informatics, Vol. 26, 2007, 17-31

[52] Otsu, N., "A Threshold Selection Method from GrayLevel Histograms," IEEE Transactions on Systems, Man, and Cybernetics, Vol. 9, No. 1, 1979, pp. 62-66.

[53] Suman Tatiraju and Avi Mehta - Image Segmentation using k-means clustering, EM and Normalized Cuts" www.ics.uci.edu/ dramanan/teaching/ics273a.../avim_re port.pdf

[54] Dunn, J. C.: A Fuzzy Relative of the ISODATA Process and its Use in Detecting Compact Well Separated Clusters. Journal of Cybernetics, Vol. 3, 1974, pp. 32 57.

[55] Bezdek, J. C.: Pattern Recognition with Fuzzy Objective Function Algorithms. New York: Plenum Press, 1981.

[56] Pr^eteux, F., Fetita, C., Capderou, A., Grenier, P.: Modeling, segmentation, and caliber estimation of bronchi in high resolution computerized tomography. Journal of Electronic Imaging 8 (1999) 36-45.

[57] Fetita, C., Preteux, F., Beigelman-Aubry, C., Grenier, P.: Pulmonary Airways: 3-D Reconstruction From Multislice CT and Clinical Investigation. IEEE Transactions on Medical Imaging 23 (2004) 1353-1364

[58] http://www.di.univr.it/documenti/OccorrenzaIns/matdid/ matdid453675.pdf

[59] https://arxiv.org/pdf/1705.08790.pdf

[60] https://www2.eecs.berkeley.edu/Research/Projects/CS/vi sion/bsds/ 